\title{
Applications industrielles des technologies supercritiques : état de l'art et perspectives
}

\author{
Gérard Charbita ${ }^{\mathrm{a}}$, Elisabeth Badens et Olivier Boutin \\ Laboratoire de Procédés Propres et Environnement, Europôle de l'Arbois, Bâtiment Laennec, BP 80, \\ 13545 Aix-en-Provence Cedex 4, France
}

Reçu le 26 septembre 2003, accepté le 18 décembre 2003

\begin{abstract}
Résumé - Les propriétés des fluides supercritiques sont à mi-chemin entre celles des gaz et celles des liquides; ces propriétés peuvent en outre être considérablement modifiées par simple variation de la pression et/ou de la température. Cette spécificité est à l'origine de nombreuses applications dans lesquelles les fluides supercritiques se présentent comme des substituts de choix aux solvants organiques traditionnels. L'extraction et la purification sont les premières applications à avoir été développées; la facilité de séparation du solvant et des solutés extraits par simple dépressurisation constitue l'atout majeur de cette méthode. Plus récemment d'autres applications très prometteuses ont émergé. Elles concernent les domaines de la réaction chimique, de la chromatographie et du traitement du solide. Initialement freinées dans leur développement par des coûts d'investissement supérieurs à ceux des procédés traditionnels, ces techniques supercritiques connaissent depuis peu un net regain d'intérêt, lié entre autres aux contraintes environnementales de plus en plus fortes pesant sur l'utilisation des solvants traditionnels.
\end{abstract}

Mots clés : Fluides supercritiques / applications industrielles / micronisation / particules composites

Abstract - Industrial applications of supercritical technologies: a review. The physico-chemical properties of supercritical fluids are halfway between those of gases and liquids; more these properties can be easily modified by a simple variation of pressure or/and temperature. It has been taken advantage of this feature to develop a lot of applications where the supercritical fluid serves as substitute for the conventional organic solvents. Extraction and purification were the first applications developed; the major advantage of this method results from the extreme easiness for separating the solvent from the solutes by a simple depressurization. More recently other attractive applications were developed that concerned two fields: chemical reaction and solid treatment. The investment costs generated by these techniques are higher than those of conventional processes and, for a long time, this has restrained their rise. However, the environmental legislation in relation to the use of solvents is noticeably evoluting, in the direction of more and more constraining instructions. This is at the origin of the obvious renewed interest which one can note today for these techniques.

Key words: Supercritical fluids / industrial applications / micronization / composite particles

\section{Introduction}

Connues depuis très longtemps [1], les propriétés des fluides supercritiques n'ont en fait que très récemment vu se développer des applications à l'échelle industrielle. C'est en 1974 que débute une longue série de brevets consacrés à la décaféination du café et du thé par le dioxyde de carbone supercritique $[2,3]$; les derniers brevets de cette liste datent de 1984. S'il est vrai que cette

\footnotetext{
a Auteur correspondant :

gerard.charbit@univ.u-3mrs.fr
}

application est désormais la plus connue, il faut cependant noter que dès 1931 Auerbach proposait un procédé de traitement d'huiles lubrifiantes par le $\mathrm{CO}_{2}$ supercritique [4]. Très rapidement, dans les années 80 , ont été proposées de nombreuses applications essentiellement axées sur l'extraction et la purification. Les perspectives entrevues à cette époque se sont révélées par trop optimistes ce qui a finalement eu pour conséquence de jeter un certain discrédit sur ces techniques, et par voie de conséquence, d'en freiner le développement [5]. Parmi les raisons objectives de ce désintérêt temporaire figure le coût d'investissement engendré par des appareillages opérant sous 
pression. En revanche, contrairement à des idées reçues, le coût de fonctionnement de ces procédés est tout à fait comparable voire inférieur à celui des procédés classiques, ainsi qu'il a été montré dans le cas de l'extraction [6].

Un autre handicap à l'essor de ces techniques réside dans le manque de données thermodynamiques et/ou hydrodynamiques qui permettraient sans doute de réaliser la transposition des résultats du laboratoire à l'échelle industrielle de façon plus déterministe. Cependant, les travaux entrepris par un grand nombre d'équipes de recherche ont permis d'identifier de nouvelles niches d'applications dans lesquelles ces techniques redeviennent concurrentielles, soit parce que les procédés conventionnels ne permettent pas d'accéder à la même qualité de résultats, ou encore parce que les surcoûts d'investissement pèsent moins lorsque les molécules traitées possèdent une forte valeur ajoutée. On peut considérer que les années 90 constituent le point de départ d'une lente reconquête s'appuyant sur une intense activité de recherche focalisée sur ces nouveaux créneaux. Comme on le verra, même si des obstacles théoriques ou technologiques restent encore à surmonter, le champ d'activité dépasse désormais très largement le domaine de l'extraction, et les perspectives sont à nouveau encourageantes. Ces perspectives s'affirment d'autant plus que d'année en année, la législation relative à l'usage des solvants devient de plus en plus contraignante; il est ainsi prévu que d'ici cinq ans l'usage de tout solvant organique sera exclu dans le domaine de l'agro-alimentaire. Dans ces conditions, le recours aux fluides supercritiques peut rapidement devenir une alternative à la fois séduisante et incontournable.

Le but de cet article est de proposer un tour d'horizon général des applications de ces techniques, qu'elles soient des réalités industrielles ou en devenir. L'étendue des travaux publiés, des brevets pris et des réalisations effectives est telle qu'il n'est pas possible ici d'être exhaustif. Dans ces conditions, outre les références citées, cet article renverra à des ouvrages ou publications plus détaillés, sur des domaines d'application plus ciblés.

Avant d'entrer dans la description des applications, il est utile de rappeler quelques définitions et propriétés des fluides supercritiques. Sans entreprendre un cours de chimie physique on peut rappeler que le diagramme d'état du corps pur décrit les différents domaines d'existence de la matière sous ses trois formes : liquide, solide et gazeuse. La figure 1 représente ce diagramme et souligne l'existence du point triple pour lequel ces trois états coexistent. Elle montre en outre que la courbe de condensationvaporisation qui constitue la frontière entre le domaine liquide et le domaine gazeux s'achève en un point dit «point critique» de coordonnées $P_{\mathrm{C}}$ et $T_{\mathrm{C}}$. Au-delà de ce point la coexistence de ces deux phases n'est plus possible et toute variation de pression ou de température se traduit par la modification des propriétés du fluide sans changement d'état. Dès lors, on a défini un domaine supercritique, celui correspondant à des températures et pressions supérieures aux coordonnées critiques; par extension le fluide est dit supercritique lorsque sa pression et sa température se situent dans ce domaine.

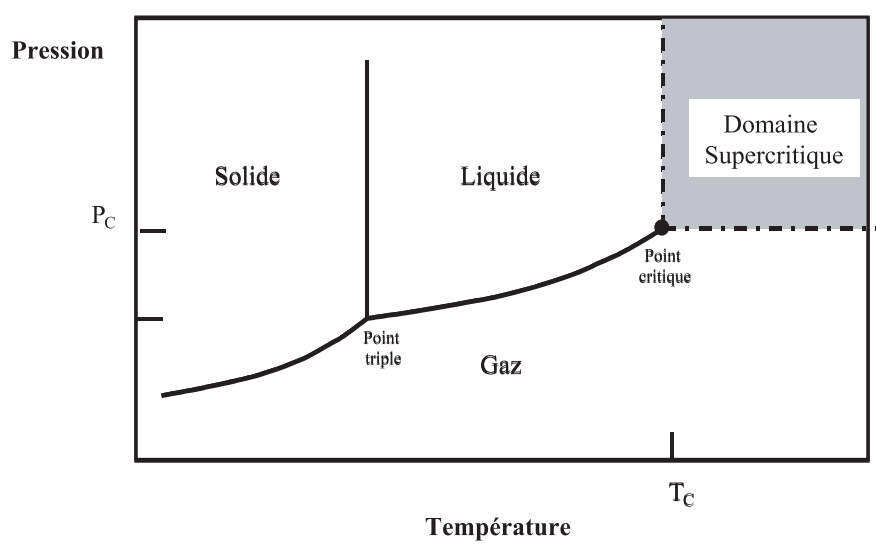

Fig. 1. Diagramme d'état du corps pur.

Une des raisons majeures à l'intérêt suscité par les fluides supercritiques réside dans la spécificité de leurs propriétés physico-chimiques qui sont un compromis entre celles des liquides et celles des gaz. Par leur diffusivité élevée et leur faible viscosité les fluides supercritiques s'apparentent aux gaz; il en résulte de bonnes aptitudes au transfert de matière. Leur masse volumique est très supérieure à celle des gaz, ce qui les rapproche plutôt des liquides en leur conférant des propriétés de solvant. Enfin, la plupart des composés utilisés dans les applications développées jusqu'ici sont gazeux dans des conditions normales de température et de pression. La conséquence en est, qu'utilisés comme solvants d'extraction, ils se séparent spontanément des solutés extraits par simple diminution de la pression. Cette spécificité est un atout majeur dans la mesure où elle permet de simplifier considérablement les procédés en dispensant des étapes de séparation des procédés conventionnels.

Il a été fait appel à de très nombreux composés lors du développement d'applications : on peut citer entre autres l'éthane, l'éthylène, le propane, ou l'ammoniac. Cependant, le composé de très loin le plus utilisé est le dioxyde de carbone $\mathrm{CO}_{2}$ car il réunit un ensemble de caractéristiques très intéressantes. Son coût est très faible, il est ininflammable, peu réactif, non toxique et gazeux dans les conditions ambiantes. Enfin ses coordonnées critiques sont peu élevées : $P_{\mathrm{C}}=7,38 \mathrm{MPa}$ et $T_{\mathrm{C}}=31,06{ }^{\circ} \mathrm{C}$, ce qui le prédestine au traitement des molécule thermolabiles sans risque excessif de dégradation. Il convient néanmoins de souligner que le dioxyde de carbone étant une molécule apolaire, son pouvoir solvant vis-à-vis des molécules polaires est réduit. Comme on le verra, cette particularité peut constituer un atout pour certaines applications et être un inconvénient pour d'autres.

\section{Les applications}

\subsection{L'extraction et la purification}

Ce sont, sans contestation possible, les applications les plus anciennes et les plus implantées au plan industriel. Elles reposent sur le fait que la supercriticité implique un état dense de la matière et confère au composé 


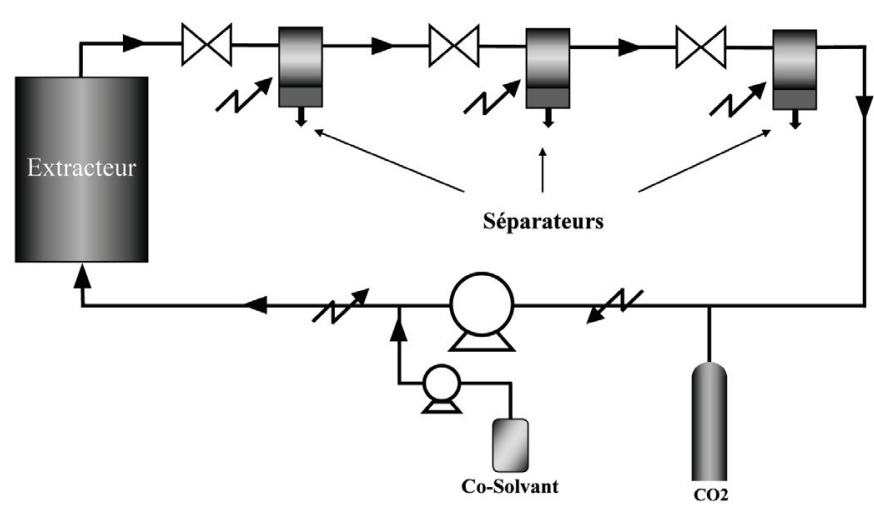

Fig. 2. Schéma d'une installation d'extraction pour des matrices solides.

considéré des propriétés de solvant. Le dioxyde de carbone peut ainsi remplacer les solvants traditionnels pour récupérer des molécules à haute valeur ajoutée ou éliminer sélectivement des impuretés. Lorsque le pouvoir solvant du $\mathrm{CO}_{2}$ s'avère inadapté vis-à-vis de la molécule visée, on additionne en faible quantité un co-solvant (parfois appelé entraîneur) afin d'ajuster la capacité de dissolution. Le schéma de procédé représenté en figure 2 illustre la technique de traitement d'une matrice solide. Le $\mathrm{CO}_{2}$ est porté à la pression et la température opératoires par une pompe et un échangeur de chaleur. Il percole à travers l'extracteur dans lequel la matière à traiter a été déposée; les solutés extraits se retrouvent dans la phase supercritique sortant de l'extracteur. Ce mélange subit alors une ou plusieurs étapes de détente, qui provoquent une diminution du pouvoir solvant du $\mathrm{CO}_{2}$ et la précipitation des solutés dans les séparateurs. Ces détentes étagées permettent donc d'effectuer un premier fractionnement grossier des solutés recueillis. Le $\mathrm{CO}_{2}$ est ensuite remis sous pression et recyclé. Ce traitement de type discontinu s'achève lorsque la matière à traiter est épuisée. Des procédés d'extraction continus existent pour le traitement des phases liquides, ils fonctionnent sur le même principe que les procédés d'extraction liquide-liquide.

Une description exhaustive des réalisations serait fastidieuse, tant elles sont nombreuses désormais. Les plus anciennes et les plus importantes en matière de tonnage de production sont celles de la décaféination du café et du thé, de traitement des houblons, ou de concentration d'alcools. Cependant, on peut trouver de très nombreux autres exemples en agroalimentaire : colorants naturels, agents texturants [7], arômes et parfums tels que vanille, citron, pomme, eucalyptus, coriandre, poivre, thym, paprika, bergamote... [8,9]. Le domaine de la diététique est concerné pour la production d'aliments délipidés [10,11], celui de la pharmacie l'est également pour la récupération de molécules à haute valeur ajoutée : drogues, antioxydants, acides gras poly-insaturés [12-14].

Le domaine de la pétrochimie est représenté par les procédés de désalphaltage des coupes pétrolières parmi lesquels le procédé ROSE $[15,16]$, et celui déjà cité du traitement des huiles lubrifiantes [4].
Le fractionnement de mélanges de polymères offre un vaste champ d'investigation dont l'appréhension reste liée à une bonne maîtrise du comportement thermodynamique complexe de ces milieux [17].

D'autres applications plus pointues mais aussi plus porteuses sont plus récemment apparues telles le séchage et la synthèse des aérogels [18], le nettoyage d'implants osseux pour la chirurgie des greffes $[19,20]$ et le déliantage des céramiques [21]. L'utilisation de fluides supercritiques pour le nettoyage de pièces de précision est une réalité depuis quelques années [22], elle s'étend désormais aux puces et circuits intégrés [23].

Enfin, la remédiation de sols contaminés est un domaine de recherche prometteur, et donc en plein expansion, cependant le transfert de ces travaux au plan industriel se heurte actuellement à des obstacles technologiques [24-26].

Un recensement des réalisations industrielles effectué en 2001 par Lack et al. [27] fait état d'une soixantaine d'unités pour une capacité globale de production d'environ 200000 t.an ${ }^{-1}$.

\subsection{La réaction chimique}

La réalité industrielle des techniques supercritiques pour la mise en œuvre de transformations chimiques est certainement moins évidente que celle de l'extraction, bien que les années 1913 à 1940 aient vu le développement de procédés tels que la synthèse de l'ammoniac, du méthanol et du polyéthylène basse densité. Il faudra attendre les années 85 pour voir la création de nouvelles unités telles que celle de synthèse du butanol par la firme japonaise Idemitsu Petrochemical Co pour une capacité de production de 40000 t.an ${ }^{-1}$ ou celle plus récente de la firme Du Pont pour la synthèse de fluoropolymères.

On distingue deux classes principales de réactions, celles réalisant la synthèse de composés, généralement de faible masse moléculaire, et celles effectuant la dégradation de polluants organiques dans les effluents aqueux.

La première de ces familles inclut de très nombreux exemples que Hutchenson a récemment récapitulés [28]. Ici encore, l'énumération de toutes les réactions étudiées serait fastidieuse. Sont cependant concernées l'hydrogénation, l'hydroformylation, l'oxydation, les cyclisations, l'isomérisation, l'alkylation, la polymérisation, l'estérification ainsi que les réactions enzymatiques. L'ensemble de ces applications exploite deux caractéristiques des milieux supercritiques :

- un pouvoir solvant aisément modulable par des variations de température et de pression. Les amplitudes de ces modifications sont d'autant plus larges que l'on se trouve dans des conditions proches de la criticité. Ainsi, parmi les grandes familles de transformations chimiques, celle de l'hydrogénation peut être citée comme exemple des améliorations potentielles qu'offrent les fluides supercritiques. Classiquement, l'hydrogénation met en présence une phase liquide, le composé à hydrogéner, une phase 
gazeuse, l'hydrogène, et un catalyseur solide. Le rendement de la transformation est limité par la faible solubilité de l'hydrogène en phase liquide et donc par la difficulté d'accès au catalyseur. En ajoutant un solvant judicieusement choisi, il est possible de former une seule phase (supercritique) dans laquelle tous les réactifs sont présents. On s'affranchit ainsi des limitations au transfert engendrées par l'existence des interfaces [29].

- La simplification de l'étape finale de séparation des solvant, produit, et catalyseur qui peut souvent se réduire à une simple dépressurisation.

La seconde famille de réaction chimique faisant l'objet de nombreuses recherches est donc celle de la dépollution des effluents aqueux. Deux variantes existent selon que l'on opère en deçà ou au-delà du point critique de l'eau $\left(P_{\mathrm{C}}=22,05 \mathrm{MPa}\right.$ et $\left.T_{\mathrm{C}}=374,2{ }^{\circ} \mathrm{C}\right)$. Avant le point critique on parle d'oxydation par voie humide, alors qu'au dessus du point critique on fait de l'oxydation hydrothermale. Dans les deux cas, la phase aqueuse est portée à température et pression opératoires, et mise en contact avec un oxydant qui peut être de l'air, de l'eau oxygénée ou de l'oxygène. Dans des conditions idéales, la combustion est complète et les polluants organiques sont décomposés pour former du dioxyde de carbone et de l'eau [30-32]. L'oxydation hydrothermale a pour principal intérêt de ne nécessiter que de très faibles temps de réaction (quelques secondes), ce qui permet de traiter de gros débits avec de petits réacteurs, alors que les temps correspondants lors d'une oxydation par voie humide sont de l'ordre de quelques minutes. À côté de cet atout, l'oxydation hydrothermale souffre d'un inconvénient important lié à la très faible solubilité des composés minéraux dans l'eau supercritique, ce qui peut conduire à leur précipitation en masse dans le réacteur; l'oxydation par voie humide est beaucoup moins handicapée par cette limitation. Il faut souligner également que la présence de composés organiques dans ces milieux réactionnels très oxydants est souvent à l'origine de problèmes aigus de corrosion des matériaux constituant les réacteurs. L'utilisation d'aciers spéciaux très élaborés, mais également coûteux, peut permettre de remédier à ce problème, mais la recherche s'oriente actuellement vers des solutions alternatives. En particulier il est fait appel à des technologies différentes telles que celle des réacteurs dans lesquels un cylindre poreux en alumine est centré dans un réacteur en acier. La réaction est alors confinée dans le cœur en alumine insensible à la corrosion [33,34].

Malgré ces inconvénients, un nombre croissant d'unités sont mises en service avec des capacités de traitement allant de quelques centaines de litres à environ $3 \mathrm{~m}^{3}$ /heure. Une liste non exhaustive inclut les installations des Sociétés Kobe Steel ltd, Hydroprocessing LLC et Ecowaste Technology.

\subsection{Le traitement du solide}

Ce domaine d'application est celui qui a émergé le plus récemment. Les brevets les plus anciens datent de
1982 [35] et sont relatifs à la mise en forme de pigments organiques. Il faudra attendre 1989 et les travaux de Gallagher et al. sur la comminution d'explosifs [36] pour que ce type d'application des technologies supercritiques soit à nouveau un sujet d'intérêt. Depuis le milieu des années 90, les recherches sur ce thème ont augmenté de façon exponentielle, elles concernent principalement, comme on le verra, le domaine de la pharmacie. On distingue ici deux familles d'applications, celle de l'imprégnation et celle réunissant la micronisation et l'encapsulation. Dans le premier cas il s'agit de déposer une molécule active sur un support solide qui peut être un polymère ou un support poreux, afin de conférer à la préparation des propriétés ciblées. Dans le second cas, il s'agit de contrôler la dissolution d'une molécule active solide. En réduisant sa taille, c'est-à-dire en la micronisant, on visera l'accélération du processus de dissolution, alors qu'en l'encapsulant ou en l'enrobant, on recherchera au contraire à retarder ou prolonger cette libération. Il est également possible d'accélérer la dissolution d'un principe actif en l'enrobant.

\subsubsection{L'imprégnation}

Ces applications mettent à profit la grande diffusivité et la faible viscosité des fluides supercritiques qui leur permettent une pénétration dans les milieux poreux notablement améliorée et plus homogène par rapport aux liquides. Dans le cas de supports polymériques, le gonflement de ces derniers en présence d'un fluide supercritique, permet également une bonne diffusion de la molécule active. L'imprégnation par fluide supercritique concerne des applications aussi diverses que le traitement du bois par des fongicides [37], la préparation de patches [38,39], de comprimés [40], l'aromatisation d'un excipient [41] ou de films polymériques, la teinture de textiles naturels ou synthétiques [42-44]. Le cas particulier des textiles montre clairement les améliorations que les techniques supercritiques sont susceptibles d'apporter. En effet la méthode traditionnelle de teinture des textiles est génératrice de grandes quantités d'eaux polluées (il faut 150 litres d'eau pour $1 \mathrm{~kg}$ de coton); ces eaux doivent donc subir un traitement avant leur rejet dans le milieu naturel. En outre, une fois teint, le textile doit subir un traitement de séchage, gros consommateur d'énergie. Le passage par les techniques supercritiques permet de s'affranchir de ces difficultés. Il faut en outre signaler que les méthodes conventionnelles ne permettent pas de teindre dans la masse certains polymères synthétiques tels que le Kevlar avec lequel sont confectionnés les vêtements de pompiers qui, faute de mieux, reçoivent une enduction peu résistante à des conditions extrêmes de température. Les techniques d'imprégnation supercritique ont fourni une réponse à ce problème [45].

Le schéma de procédé en figure 3 illustre la simplicité du procédé supercritique. La molécule active à déposer est placée dans le saturateur et le support à imprégner dans le réacteur. Le dioxyde de carbone de la réserve est mis en pression et envoyé dans le saturateur dans lequel 


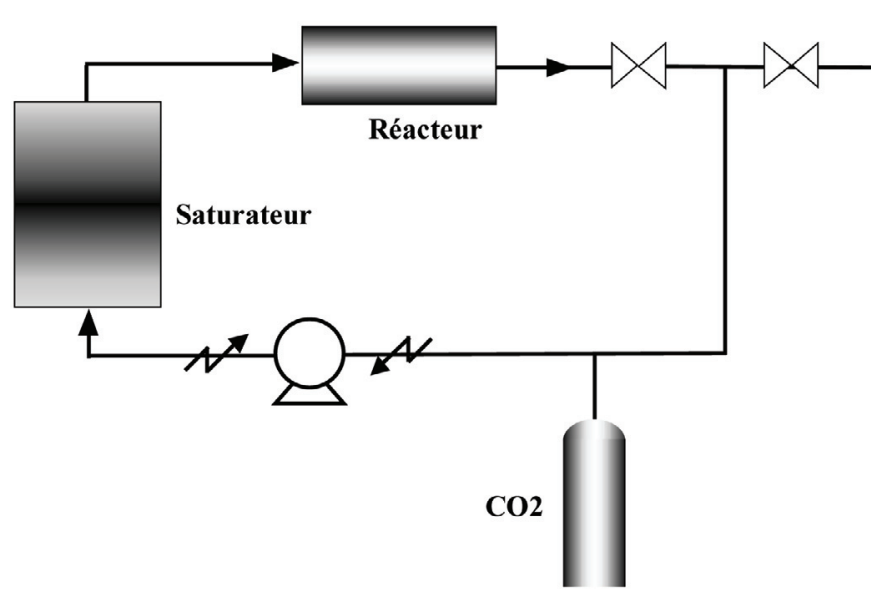

Fig. 3. Schéma d'une installation destinée à l'imprégnation.

il se charge en principe actif; il percole ensuite à travers le réacteur où il se dépose sur le support. Le mélange supercritique issu du réacteur est recyclé et la réserve ne sert donc que d'appoint. Les deux paramètres importants de ce procédé sont donc la solubilité du principe actif dans le $\mathrm{CO}_{2}$ puis son coefficient de partage entre la phase supercritique et le support.

\subsubsection{La micronisation}

La diminution de taille de particules est recherchée chaque fois que l'on souhaite, en augmentant la surface spécifique du solide, accroître son activité. Ce type d'opération peut être envisagé pour des pigments de peinture, des encres, des catalyseurs ou en galénique. C'est certainement ce dernier secteur d'activité qui jusqu'ici a le plus retenu l'attention des équipes de recherche. En effet la micronisation accélère la dissolution des médicaments dans le tractus gastro-intestinal et augmente par là même leur bio-disponibilité. Par ailleurs des particules de faible dimension $(5 \mu \mathrm{m})$ peuvent permettre des modes d'administration par aérosol beaucoup moins contraignants que les injections sous-cutanées. Les méthodes conventionnelles les plus connues permettant de microniser un solide sont le broyage, la micronisation par jet d'air. Cependant, ces méthodes présentent pour chacune d'entre elles une ou plusieurs limitations parmi lesquelles des surchauffes locales, des tailles finales trop importantes ou des répartitions de taille trop larges. Les technologies supercritiques sont à même de proposer des solutions pour pallier ces inconvénients; on peut schématiquement répartir ces technologies en deux classes principales.

\subsubsection{Le solide est soluble dans le fluide supercritique}

Le procédé utilisé ici est le procédé RESS (Rapid Expansion of Supercritical Solutions), décrit en figure 4. C'est la technique la plus simple : le fluide supercritique est envoyé sous pression dans un autoclave initialement chargé en solide, où il dissout le solide ainsi entraîné dans la phase supercritique. Celle-ci est soumise à une
$\mathrm{CO} 2$

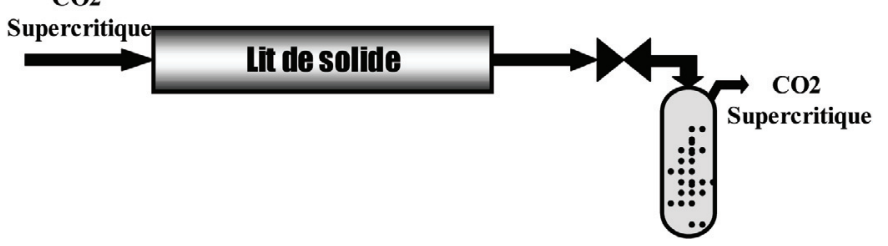

Fig. 4. Micronisation par le procédé RESS : schéma d'installation.

Tableau 1. Exemples de molécules à usage pharmaceutique micronisées par le procédé RESS.

\begin{tabular}{lll}
\hline Molécule & Fluide supercritique & Référence \\
\hline Acide benzoïque & $\mathrm{CO}_{2}$ & Tavana [46] \\
$\beta$-carotène & $\mathrm{C}_{2} \mathrm{H}_{4}+$ Toluène & Chang [47] \\
Cholesterol & $\mathrm{CO}_{2}, \mathrm{CHF}_{3}$ & Helfegen [48] \\
Cyclosporine & $\mathrm{CO}_{2}$ & Pace [49] \\
Fenofibrate & $\mathrm{CO}_{2}$ & Pace [49] \\
Griséofulvine & $\mathrm{CHF}_{3}$ & Reverchon [50] \\
Lovastatine & $\mathrm{CO}_{2}$ & Larson [51] \\
Naproxène & $\mathrm{CO}_{2}$ & Kim [52] \\
Progestérone & $\mathrm{CO}_{2}$ & Coffey [53] \\
Acide salicylique & $\mathrm{CO}_{2}$ & Reverchon [54] \\
\hline
\end{tabular}

rapide diminution de pression par le biais d'un dispositif déprimogène : orifice ou capillaire. Cette chute de pression provoque une diminution du pouvoir solvant $\mathrm{du}$ fluide supercritique et crée une sursaturation en soluté qui précipite. Cette méthode est séduisante car elle ne fait appel à aucun solvant organique et produit donc des particules d'une extrême propreté. En outre ces particules sont fines (jusqu'à quelques dizaines de nanomètres) et la répartition des tailles très serrée. Le principal inconvénient de cette méthode est lié à la faible solubilité de nombreux médicaments dans le dioxyde carbone supercritique, ce qui nécessite d'opérer généralement sous hautes pressions (plusieurs dizaines de $\mathrm{MPa}$ ), ou de changer la nature du fluide supercritique. Malgré cette limitation, de nombreuses molécules ont été traitées par cette technique, le tableau 1 donne un aperçu volontairement limité de la diversité de ces travaux dans le domaine de la pharmacie. Cette technique a également été mise en œuvre avec succès pour traiter des polymères ou des composés aromatiques tels que la vanilline.

\subsubsection{Le solide n'est pas soluble dans le fluide supercritique : les techniques antisolvant}

Dans ce cas de figure, on utilise une méthode bien connue des chimistes qui consiste dans un premier temps à dissoudre le solide dans un premier solvant liquide; on additionne ensuite à cette solution un second solvant très soluble dans le premier mais dans lequel le solide n'est pas soluble. Cette addition provoque une sursaturation dans la phase liquide et la précipitation du solide; le second solvant joue donc le rôle d'un antisolvant par rapport au soluté qui cristallise. Il y a donc un effet combiné d'antisolvant et d'évaporation du solvant. S'agissant des technologies supercritiques, le principe de la méthode 


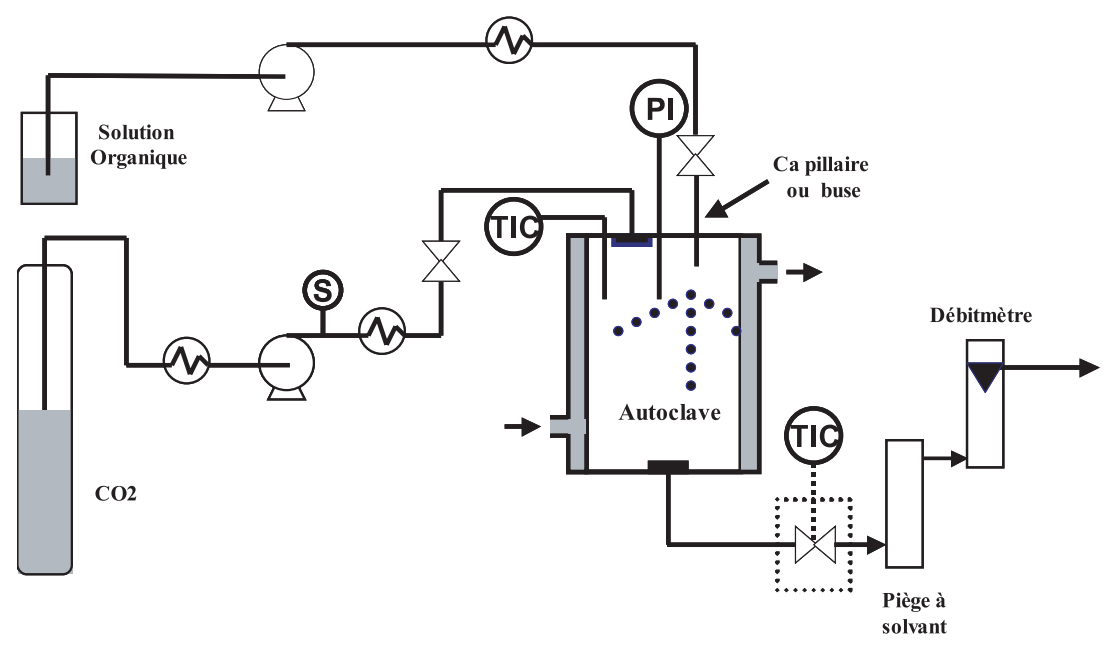

Fig. 5. Micronisation par le procédé SAS : schéma d'installation.

est identique, la seule différence avec le mode opératoire précédent étant que le second solvant est un fluide supercritique au lieu d'être un liquide. Différentes variantes existent, elles différent par le mode de mise en contact de la phase liquide et de la phase supercritique.

Dans la procédure dite GAS (Gas AntiSolvent), on introduit dans un autoclave, à pression atmosphérique, la solution liquide contenant le soluté dissous. Le fluide supercritique est ensuite dispersé progressivement dans le réacteur jusqu'à la pression de travail, provoquant la précipitation du solide. On travaille ici à des niveaux de pression compris entre 8 et $15 \mathrm{MPa}$, c'est-àdire très inférieurs à ceux du RESS. La pression finale, la température, le profil de montée en pression, sont autant de paramètres opératoires permettant de modifier la taille des particules. L'inconvénient de cette méthode de type discontinu est que la pression opératoire dans l'autoclave varie dans le temps ce qui pourrait constituer un obstacle pour une transposition à l'échelle industrielle. Il faut signaler en outre que la dissolution du fluide supercritique dans la phase liquide provoque une expansion volumique de cette dernière qui peut atteindre $1000 \%$ en fonction des conditions opératoires choisies. Ceci constitue une limitation au volume de solution liquide que l'on peut initialement introduire dans le réacteur et donc traiter lors d'un batch.

Dans la procédure SAS (Supercritical AntiSolvent), on établit dans le réacteur un courant de fluide supercritique à débit, température et pression fixés. Dans un second temps on disperse de façon continue la phase liquide dans ce continuum par l'intermédiaire d'un capillaire ou d'une buse. Les transferts de matière entre les deux phases conduisent à la précipitation du solide qui reste dans le réacteur, tandis que le solvant primaire et le fluide supercritique sont évacués de façon continue. L'opération se déroule ici à pression, débit et température constants, ce qui, d'une part, autorise un meilleur contrôle de la taille des particules formées, et qui, d'autre part, est plus favorable pour une transposition à l'échelle industrielle. Les paramètres opératoires permettant d'influer sur la taille des particules sont ici plus nombreux que pour la technique GAS. Outre la température et la pression, les débits des phases liquide et supercritique jouent un rôle considérable de même que la nature et la taille du dispositif de dispersion. La figure 5 représente un schéma d'installation pour le procédé SAS.

Une variante de la technique SAS est constituée par le procédé SEDS (Solution Enhanced Dispersion by Supercritical Solutions). La différence essentielle entre les deux variantes tient à la nature du dispositif d'injection des deux phases fluides. Contrairement au SAS dans lequel les dispositifs d'introduction des deux phases sont distincts, dans le SEDS les deux phases sont introduites par une buse coaxiale, ce qui devrait améliorer les conditions hydrodynamiques de leur mélange et conduire à des transferts de masse accélérés donc à des particules plus fines et plus homogènes en taille.

La grande souplesse des procédés antisolvants leur vaut d'avoir été testés avec succès pour une très large gamme de produits, ce que ne reflète pas le tableau 2 volontairement restrictif; un choix plus large de référence est fourni par les articles de Jung et al. [74] et Charbit et al. [75].

\subsubsection{Les particules composites}

Ainsi qu'il est dit plus haut, la génération de particules composites a pour principe d'associer un excipient à une molécule active afin de modifier les propriétés de cette dernière. Dans la grande majorité des travaux rapportés dans la littérature, l'excipient a pour effet de permettre le contrôle de la libération du principe actif en empêchant une dissolution trop rapide de ce dernier ; on parle alors de système à effet retard. La libération de la molécule active depuis la particule a lieu par l'un des processus suivants : elle diffuse progressivement à travers une couche externe constituée d'excipient, ou encore la couche externe d'excipient se délite progressivement libérant ainsi le principe actif. Les exemples rapportés visent essentiellement des molécules à usage thérapeutique [56,76-80], mais il a été 
Tableau 2. Exemples de molécules à usage pharmaceutique micronisées par les procédés antisolvants.

\begin{tabular}{lclll}
\hline \multicolumn{1}{c}{ Molécule } & Procédé & \multicolumn{1}{c}{ Solvant } & Antisolvant & \multicolumn{1}{c}{ Référence } \\
\hline Acétaminophène & GAS & Ethanol & $\mathrm{CO} 2$ & Wubbolts [55] \\
Carbamazépine & GAS & Acétone & $\mathrm{CO} 2$ & Moneghini [56] \\
Chlorpropamide & GAS & Acétone & $\mathrm{CO} 2$ & Yeo [57] \\
Insuline & GAS & Diméthyl sulfoxyde & $\mathrm{CO} 2$ & Yeo [58] \\
Insuline & GAS & Méthanol & $\mathrm{CO} 2$ & Thiering [59] \\
Sulfothiazole & GAS & Ethanol & $\mathrm{CO} 2$ & Kitamura [60] \\
Amoxicilline & SAS & N-méthyl pyrrolidone & $\mathrm{CO} 2$ & Reverchon [61] \\
Griséofulvine & SAS & Chlorure de méthylène & $\mathrm{CO} 2$ & Reverchon [62] \\
Hydrocortisone & SAS & Diméthyl sulfoxyde & $\mathrm{CO} 2$ & Said [63] \\
Méthylprédnilosone & SAS & Tétrahydrofurane & $\mathrm{CO} 2$ & Sze Tu [64] \\
Phospholipides & SAS & Ethanol & $\mathrm{CO} 2$ & Badens [65] \\
Phospholipides & SAS & Ethanol & $\mathrm{CO} 2$ & Magnan [66] \\
Rifampicine & SAS & Diméthyl sulfoxyde & $\mathrm{CO} 2$ & Reverchon [67] \\
Aspartame & SEDS & Méthanol & $\mathrm{CO} 2$ & Hanna [68] \\
Ibuprofène & SEDS & Méthanol & $\mathrm{CO} 2-\mathrm{N}_{2}$ & Hanna [69] \\
Insuline & SEDS & Acetate d'ethyl & $\mathrm{CO}{ }_{2}-\mathrm{N}_{2}$ & Ghaderi [70] \\
Lysozyme & SEDS & Ethanol & $\mathrm{CO} 2$ & Sloan [71] \\
Maltose & SEDS & Eau & $\mathrm{CO} 2$ & Hanna [72] \\
Acide nicotinique & SEDS & Ethanol & $\mathrm{CO} 2$ & Hanna [73] \\
RhDNase & SEDS & Ethanol & $\mathrm{CO} 2$ & Hanna [68] \\
\hline
\end{tabular}

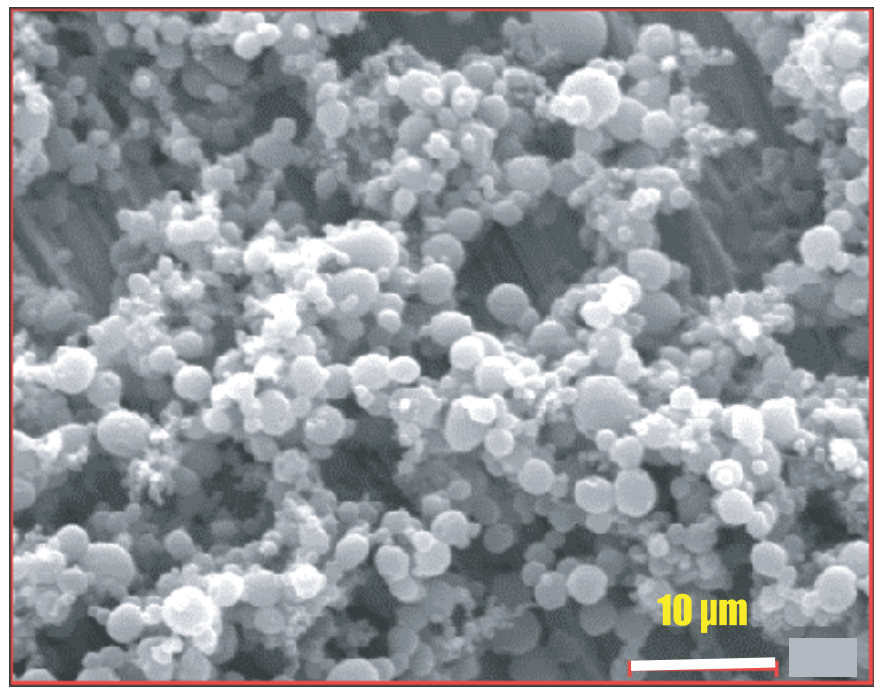

Fig. 6. Cliché MEB de particules composites de diuron et d'acide (l-polylactique).

montré que la co-précipitation d'herbicide et de polymère était également possible [81]. La figure 6 est un cliché MEB de particules composites formées de diuron, un herbicide très utilisé, et de L-PLA (acide L-polylactique). La figure 7 illustre clairement l'effet retard, puisque dans des conditions identiques, la totalité du diuron non traité est dissoute dans l'eau en quelques heures, alors que pour les particules composites polymère-diuron, il faut 20 jours pour en libérer $40 \%$.

Parallèlement à ces travaux visant un effet retard, on a vu plus récemment apparaître des travaux relatifs à l'association d'un complexant et d'un principe actif afin d'augmenter la bio-disponibilité de ce dernier. De très nombreuses molécules thérapeutiques sont peu hydrosolubles,

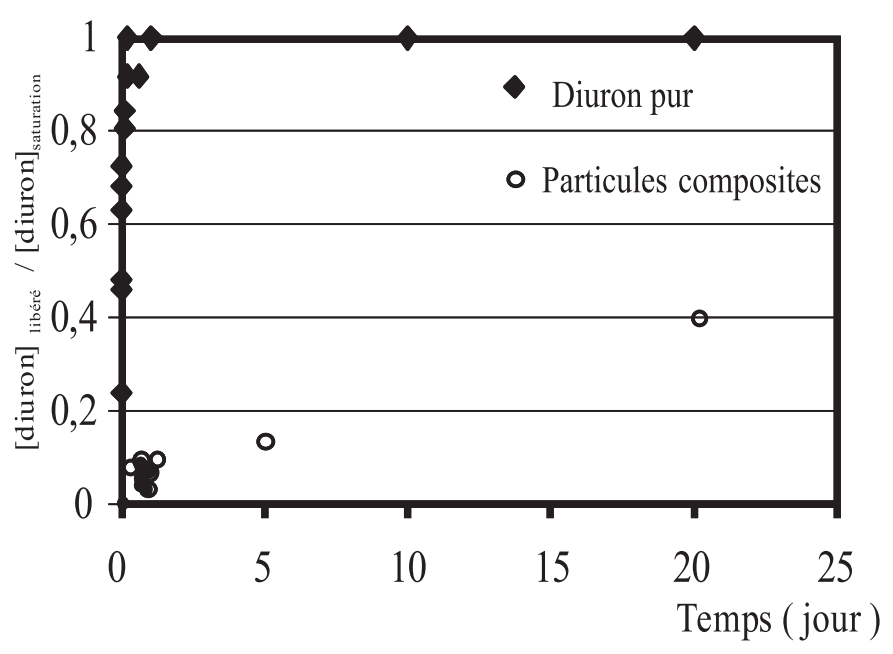

Fig. 7. Effet retard : cinétiques de dissolution de particules de diuron brut et de particules composites.

et sont éliminées de l'organisme avant d'avoir traversé les parois gastriques et intestinales. En associant ces molécules à des composés tels que les cyclodextrines ou certains polymères, il a été possible de masquer leur hydrophobicité, d'accélérer leur dissolution et d'améliorer considérablement leur bio-disponibilité [82].

Les méthodes expérimentales mises en œuvre pour préparer ces particules composites sont analogues à celles décrites plus haut pour la pratique de la micronisation. Dans le cas du procédé SAS, pour ne citer que cet exemple, la différence essentielle avec ce qui précède réside dans le fait que la solution liquide qui sera dispersée est désormais composée du principe actif, de l'excipient et du solvant organique.

Ainsi qu'il a été dit plus haut, ce domaine d'application est celui qui a été exploré le plus récemment, de sorte 
qu'à l'heure actuelle, aucune réalisation industrielle n'a été annoncée. Cependant, le rachat de petites structures de recherche par des grands groupes pharmaceutiques, ainsi que le nombre sans cesse croissant de prise de brevets, sont les signes tangibles d'une potentialité qui se traduira immanquablement par des réalisations industrielles dans un futur proche. Le contrôle du polymorphisme, réalisable par ces techniques, est également un point clé pour les industries pharmaceutiques. Ainsi de façon paradoxale, il apparaît que parmi les applications possibles des technologies supercritiques, la moins développée est sans doute la plus prometteuse.

\section{Les limitations}

Une idée couramment répandue dans le monde industriel est que les technologies supercritiques sont beaucoup plus onéreuses que les technologies classiques du fait de coûts d'investissement excessifs; ceci constitue un frein néfaste à leur développement. Si ce sentiment est fondé pour de petites installations, il s'avère loin de la vérité lorsque les capacités des installations grandissent ainsi que l'ont démontré les réalisations dédiées à la décaféination du café par exemple. En réalité, comme l'a montré Perrut [83], les coûts d'investissement croissent lentement avec la capacité et il faut préciser en outre que les coûts de fonctionnement sont peu élevés.

Certains problèmes technologiques non résolus constituent également des freins à l'essor de ces méthodes. Ainsi, l'usage des fluides supercritiques pour procéder à la remédiation de terres contaminées s'avère d'une grande efficacité. Cependant, le traitement de grandes quantités de matière ne peut se concevoir qu'à travers un procédé fonctionnant en continu, ce qui bute sur le problème technologique suivant : on ne sait pas alimenter en solide et de façon continue, un réacteur fonctionnant sous pression.

Un autre exemple de frein technologique est celui évoqué plus haut dans le cas de l'oxydation hydrothermale. La dégradation des matériaux occasionnée par la corrosion nécessite des solutions alternatives pour la conception des réacteurs; il semble qu'aucune de celles proposées jusqu'ici ne fasse l'unanimité.

Le dernier obstacle au transfert industriel des travaux des équipes de recherche est celui du manque de connaissances fondamentales dans des domaines de la thermodynamique et de l'hydrodynamique. Pour ne s'en tenir qu'au seul exemple de la micronisation par le procédé SAS décrit plus haut, il est clair que si l'on ne connaît pas le comportement (diagramme de phases) du ternaire solutésolvant-antisolvant, en fonction de la température, de la pression et des concentrations, il est difficile de prédire la nature ou la taille des particules formées. Ces diagrammes représentent un travail lourd et complexe comme toute expérimentation en condition de haute pression.

De la même façon, la littérature ne recèle que très peu de travaux sur la dispersion d'une phase liquide dans un continuum supercritique. Il est donc impossible actuellement de prévoir la taille des gouttes formées en sortie de buse, or cette taille conditionne de façon significative l'intensité des échanges de matière entre la phase liquide et la phase supercritique. Là encore l'expérimentation s'avère lourde et difficile.

Ces limitations, par manque de connaissances fondamentales, constituent un handicap pour la réalisation d'une transposition d'échelle, et dans certains cas l'extrapolation peut relever d'un semi-empirisme avec les risques inhérents. Il reste donc beaucoup de travail en perspective afin de faire reposer l'extrapolation sur des bases plus objectives. Fort heureusement, les applications de traitement du solide et plus particulièrement celles de la micronisation et de l'encapsulation qui sont les plus attractives, relèvent de secteurs d'activité tels que la galénique, où les tonnages de production sont faibles. Dans ces conditions les tailles de réacteurs pour une installation industrielle restent modestes et de ce fait les transpositions moins hasardeuses.

\section{Conclusion et perspectives}

On peut aujourd'hui considérer que certaines applications telles que l'extraction et la purification sont des techniques matures si l'on se réfère au savoir accumulé et au nombre d'installations existantes. D'autres plus juvéniles et en émergence n'ont pas encore véritablement trouvé leur place malgré les promesses engendrées par les résultats des recherches menées dans les laboratoires. S'il est vrai que les techniques supercritiques ne constituent pas systématiquement la meilleure alternative, il faut également souligner qu'elles ont dans certains cas offert des solutions que n'étaient pas susceptibles de fournir les voies conventionnelles. En outre, et d'une façon générale, la qualité des produits finis obtenus par voie classique est inférieure, ce qui constitue un atout indéniable. Le label « chimie verte», attaché aux technologies supercritiques constitue désormais un argument de marketing auquel les clients potentiels sont sensibles. Enfin, ainsi qu'il a été évoqué plus haut, la législation déjà lourde, qui définit le cadre d'utilisation des solvants organiques, va devenir de plus en plus pesante au point que dans certains domaines tels que celui de l'agro-alimentaire, ils seront totalement bannis. Cette perspective à court terme, ne peut aller que dans le sens d'un recours sans cesse croissant à ces nouvelles approches et concourir définitivement à leur essor.

\section{Références}

[1] J.B. Hannay, J. Hogarth, On the solubility of solids in gases, Proceedings of the Royal Society of London, 29, 1879 , p. 324

[2] K. Zosel, Process for recovering caffeine, U.S. Patent 3 806 619, Avril 1974

[3] K. Zosel, Process for the direct decaffeination of aqueous coffee extract solutions, U.S. Patent 4348422 (Sep. 7, 1982) 
[4] E.B. Auerbach, Process for treating, separating, and purifying oils, U.S. Patent 1805751 (1931)

[5] V. Krukonis, G. Brunner, M. Perrut, Industrial operations with supercritical fluids : current processes and perspectives of the future. Proceedings of the 3rd Intenational Symposium on Supercritical Fluids, Strasbourg, 1994, vol. 1, p. 1

[6] P. Pellerin, Comparing extraction by traditional solvents with supercritical extraction: An economic and environmental standpoint, Proceedings of the 6th International Symposium on Supercritical fluids, Versailles, 2003, pp. $11-15$

[7] M. Perrut, Supercritical fluid applications: Industrial developments and economic issues, in Eng. Chem. Res. 39 (2000) 4531-4535

[8] T. Gamse, I. Rogler, R. Marr, Use of supercritical fluids for different processes including new developments: A review, Chem. Eng. and Proc. 39 (2000) 19-28

[9] K. Udaya Sankar, Supercritical fluid carbon dioxide technology for extraction of spices and other high value bioactive compounds, dans Supercritical Fluid Processing of Food and Biomaterials, Chapman and Hall, 1994, pp. $155-167$

[10] A. Castera, Production of low-fat and low-cholesterol foodstuffs or biological products by supercritical $\mathrm{CO}_{2}$ extraction: processes and applications, dans Supercritical Fluid Processing of Food and Biomaterials, Chapman and Hall, 1994, pp. 187-201

[11] S. Vijayan, D.P. Byskal, L.P. Buckley, Separation of oil from fried chips by a supercritical extraction process: An overview of bench-scale experience and process economics, dans Supercritical Fluid Processing of Food and Biomaterials, Chapman and Hall, 1994, pp. 75-92

[12] U. Nguyen, D.A. Evans, G. Frakman, Natural antioxidants produced by supercritical extraction, dans Supercritical Fluid Processing of Food and Biomaterials, Chapman and Hall, 1994, pp. 103-113

[13] G.B. Guarise, A. Bertucco, P. Pallado, Carbon dioxide as a supercritical solvent in fatty acid refining: Theory and practice, dans Supercritical Fluid Processing of Food and Biomaterials, Chapman and Hall, 1994, pp. 27-43

[14] G. Brunner, S. Peter, On the solubility of glycerides and fatty acids in compressed gases in presence of an entrainer, Sep. Sci. and Tech. 17 (1982) 199-214

[15] J.A. Gearhart, L. Garwin, ROSE process improves resid feed, Hydrocarbon Process. 55 (1976) 125-128

[16] R.E. Wilson, P.C. Keith, R.E. Haylett, 1936. Liquid propane: Use in dewaxing, deasphalting, and refining heavy oils, Ind. Eng. Chem. 28 (1936) 1065

[17] M. Mc Hugh, V. Krukonis, Supercritical extraction, Butterworth-Heinemann, 1994, pp. 189-284

[18] L. van Ginneken, R. van de Leest, L. Willems, K. Elst, H. Weyten, Synthesis, characterisation and applications of silica aerogels, Proceedings of the 6th International Symposium on Supercritical Fluids, Versailles, 2003, pp. $1971-1976$

[19] J. Fages, A. Marty, C. Delga, J.S. Condoret, D. Combes, P. Frayssinet, Biomaterials 15 (1994) 650-656

[20] J. Fages, E. Jean, P. Frayssinet, D. Mathon, B. Poirier, A. Autefage, D. Larzul, Bone allografts and supercritical Processing; effects on osteointegration and viral safety, J. Supercritical Fluids 13 (1998) 351-356
[21] T. Chartier, F. Bordet, E. Delhomme, J.F. Baumard, Extraction of binders from green ceramic bodies by supercritical fluids: Influence of porosity, J. Europ. Ceramic Soc. 22 (2002) 1403-1409

[22] D. Lansberry, T.G. Council, Supercritical recirculating system for a precision inertial instrument cleaner, J. Cleaner Production 4 (1996) 133-134

[23] H. Wang, M. Hirahara, M. Goto, T. Tsutomu Hirose, Extraction of flame retardants from electronic printed circuit board by supercritical carbon dioxide, J. Supercritical Fluids (2003), sous presse

[24] S.B. Hawthorne, Y. Yang, D.J. Miller, Extraction of organic pollutants from environmental solids with sub and supercritical, Water. Anal. Chem. 66 (1994) 2912-2920

[25] K. Ken Li, M. Michael Landriault, M. Merv Fingas, M. Maria Ljompart, Accelerated solvent extraction (ASE) of environmental organic compounds in soils using a modified supercritical fluid extractor, J. Hazardous Materials 102 (2003) 93-104

[26] R. John, J.R. Dean, G. Guohua Xiong, Extraction of organic pollutants from environmental matrices: Selection of extraction technique, Trends in Analytical Chemistry 19 (2000) 553-564

[27] E. Lack, T. Gamse, R. Marre, Separation operations and equipment, dans High Pressure Process Technology: Fundamentals and Applications, Elsevier, 2001, pp. 351-402

[28] K.W. Hutchenson, Organic chemical reactions and catalysis in supercritical fluid media, dans Supercritical Fluid Technology in Materials Science and Engineering, M. Dekker (ed.), 2002, 87-188

[29] M. Härröd, S. van den Hark, A. Holmqvist, P. Moller, Hydrogenation at supercritical single phase conditions, Proceedings of the 6th International Symposium on Supercritical Fluids, Versailles, 2003, pp. 1097-1102

[30] C. Aymonier, C. Beslin, C. Jolivalt, F. Cansell, Hydrothermal oxidation of a nitrogen-containinig compound: The funuron, J. Supercritical Fluids 17 (2000) 45-54

[31] J.C. Meyer, P.A. Marone, J.W. Tester, Acetic acid oxidation and hydrolysis in supercritical water, AIChEJ. 41 (1995) 2108-2121

[32] A. Shanableh, Y. Shinuzu, Treatment of sewage sludge using hydrothermal oxidation technology application challenges, Water Sci. Technol. 41 (2000) 84-92

[33] E. Fauvel, C. Joussot-Dubien, E. Pomier, P. Guichardon, G. Charbit, F. Charbit, S. Sarrade, Modeling of a porous reactor for supercritical water oxidation by a residence time distribution study, Ind. Eng. Chem. Res. 42 (2003) 2122-2130

[34] E. Fauvel, C. Joussot-Dubien, P. Guichardon, G. Charbit, F. Charbit, S. Sarrade, A double-wall reactor for hydrothermal oxidation with supercritical water flow across the inner porous tube, J. Supercritical Fluids (2003), sous presse

[35] F. Graser, G. Wickenhaeuser, Conditioning of finely divided crude organic pigments. US Patent 4451654 (1982)

[36] P.M. Gallagher, M.P. Coffey, V.J. Krukonis, N. Klasutis, Gas antisolvent recrystallization: New process to recrystallize compounds insoluble in supercritical fluids dans Supercritical Fluid Science and Technology, ACS Symp. Ser. 406 (1989) 334-354 
[37] S. Iversen, T. Tommy Larsen, O. Ole Henriksen, K. Felsvang, The world's first commercial supercritical wood treatment plant, Proceedings of the 6th International Symposium on Supercritical Fluids, Versailles, 2003, pp. $445-450$

[38] O. Guneya, A. Akgerman, Synthesis of controlled-release products in supercritical medium AIChE J. 48 (2002) 856-866

[39] P. Alessi, A. Cortesi, I. Kikic, Effect of operating parameters on the impregnation of polymers with drugs, Proceedings of the 5th Meeting on Supercritical Fluids, Nice, 1998 , pp. $373-378$

[40] C. Magnan, C. Bazan, F. Charbit, J. Joachim, G. Charbit, Impregnation of porous supports with active substances by means of supercritical fluids, dans High Pressure Chemical Engineering, P.R. Von Rohr, C. Trepp (ed.), 1996, pp. 509-514

[41] W. Majewski, M. Perrut, On-line direct impregnation of natural extracts, Proceedings of the 7th Meeting on Supercritical Fluids, Antibes, 2000, pp. 779-780

[42] M.R. De Giorgi, E. Cadoni, D. Maricca, A. Piras, Dyeing polyester fibres with disperse dyes in supercritical $\mathrm{CO}_{2}$, Dyes and Pigments 45 (2000) 75-79

[43] T. Ngo, C.L. Liotta, C.A. Eckert, S.G. Kazarian, Supercritical fluid impregnation of different azo-dyes into polymer: in situ UV/Vis spectroscopic study, J. Supercritical Fluids 27 (2003) 215-222

[44] E. Bach, E. Cleve, E. Schollmeyer, Treatment of textiles in supercritical $\mathrm{CO}_{2}$ : New results, Proceedings of the 7th Meeting on Supercritical Fluids, Antibes, 2000, pp. $385-388$

[45] J.J. Shim, J.H. Choi, J.H. Ju, B.K. Son, J.M. Ahn, B.H. Kim, K.S. Kim, Dyeing of polyester, aramid, and polypropylene fibers in supercritical $\mathrm{CO}_{2}$, Proceedings of the 6th International Symposium on Supercritical fluids, Versailles, 2003, pp. 2101-2106

[46] A. Tavana, A.D. Randolph, Manipulating solids CSD in a supercritical fluid crystallizer: $\mathrm{CO}_{2}$ - benzoïc acid, AIChE J. 35 (1989) 1625-1630

[47] C.J. Chang, A.D. Randolph, Precipitation of micronsize organic particles from supercritical fluids, AIChE J. 35 (989) 1876-1882

[48] B. Helfegen, M. Türk, K. Schaber, Theoretical and experimental investigation of the micronization of organic solids by rapid expansion of supercritical solutions, Powder Technology 110 (2000) 22-28

[49] G. Pace, K. Mishra, B. Henriksen, V.J. Krukonis, A. Godinas, Processes to generate submicron particles of water - insoluble compounds, International Patent WO 99/65469, 1999

[50] E. Reverchon, G. Della Porta, R. Taddeo, P. Pallado, A. Stassi, Solubility and micronization of griseofulvin in supercritical $\mathrm{CHF}_{3}$, Industrial and Engineering Chemistry Research 34 (1995) 4087-4091

[51] K.A. Larson, M.L. King, Evaluation of Supercritical Fluid Extraction in the Pharmaceutical Industry, Biotechnology Progress 2 (1986) 73-82

[52] J. Kim, T. Paxton, D. Tomasko, Microencapsulation of naproxen using rapid expansion of supercritical solutions, Biotechnology Progress 12 (1996) 650-661

[53] M.P. Coffey, V. Krukonis, Supercritical Fluid Nucleation, An improved Ultrafine Particle Formation Process, Phasex Corp., Final report to NSF, 1988
[54] E. Reverchon, G. Donsi, D. Gorgoglione, Salicylic acid solubilization in supercritical $\mathrm{CO}_{2}$ and its micronization by RESS, J. Supercritical Fluids 6 (1993) 241-248

[55] F.E. Wubbolts, C. Kerach, G.M. Van Rosmalen, Semi batch precipitation of acetaminophen from ethanol with liquid carbon dioxide at a constant pressure, Proceedings of the 5th Meeting on Supercritical Fluids, Nice, 1, 1998, pp. 249-255

[56] M. Moneghini, I. Kikic, D. Voinovich, B. Perissutti, Filipovic-Grcic J., Processing of carbamazepine-PEG 4000 solid dispersions with supercritical carbon dioxide : preparation, characterization, and in vitro dissolution, Int. J. Pharmaceutics 222 (2001) 129-138

[57] S.D. Yeo, M.S. Kim, J.C. Lee, Recrystallization of sulfothiazole and chlorpropamide using the supercritical fluid antisolvent process, J. Supercritical Fluids 25 (2003) 143154

[58] S.D. Yeo, G. Lim, Debenedetti P.G., Bernstein H., Formation of microparticulate protein powders using a supercritical fluid antisolvent, Biotechnology and Bioengineering 41 (1993) 341-346

[59] R. Thiering, D. Dehghani, A. Dillow, N.R. Foster, The influence of operating conditions on the dense gas precipitation of model proteins, J. Chem. Tech. Biotech. 75 (2000) 29-41

[60] M. Kitamura, M. Yamamoto, Y. Yoshinaga, H. Masuoka, Crystal size control of sulfathiazole using high pressure carbon dioxide, J. Crystal Growth 178 (1997) 378-386

[61] E. Reverchon, I. De Marco, C. Caputo, G. Della Porta, Pilot scale micronization of amoxicillin by supercritical antisolvent precipitation, J. Supercritical Fluids 26 (2003) $1-7$

[62] E. Reverchon, G. Della Porta, Production of antibiotic micro- and nano-particles by supercritical antisolvent precipitation, Powder Technology 106 (1999) 23-29

[63] S. Said, R.A. Rajewski, V. Stella, B. Subramanian, World Patent WO 9731 691, 1997

[64] L. Sze Tu, F. Dehghani, N.R. Foster, Micronisation and microencapsulation of pharmaceuticals using a carbon dioxide antisolvent, Powder Technology 126 (2002) 134149

[65] E. Badens, C. Magnan, G. Charbit, Microparticles of soy lecithin formed by supercritical processes, Biotechnology and Bioengineering 72 (2001) 194-204

[66] C. Magnan, E. Badens, N. Commenges, G. Charbit, Soy lecithin micronization by precipitation with a compressed fluid antisolvent influence of process parameters, J. Supercritical Fluids 19 (2000) 69-77

[67] E. Reverchon, I. De Marco, G. Della Porta, Rifampicin microparticles production by supercritical antisolvent precipitation, Int. J. Pharmaceutics 243 (2002) 83-91.

[68] M. Hanna, P. York, Particle formation method and their products, US Patent 2002/0073511 A1, 2002

[69] M. Hanna, P. York, Method and apparatus for the formation of particles. European Patent WO 99/59710, 1999

[70] R. Ghaderi, P. Artursson, J. Carlfors, A new method for preparing biodegradable microparticles and entrapment of hydrocortisone in DL-PLG microparticles using supercritical fluid, Europ. J. Pharmaceutical Sciences 10 (2000) $1-9$

[71] R. Sloan, M.E. Hollowood, G.O. Humphreys, W. Ashraf, P. York, Supercritical Fluid Processing: preparation of stable protein particles. Proceedings of the 5th Meeting on Supercritical Fluids, Nice, 1998, pp. 301-306 
[72] M.H. Hanna, P. York, Method and apparatus for the formulation of particles. WO 96/00 610, 1996

[73] M.H. Hanna, P. York, Method and apparatus for the formation of particles, European Patent WO 98/36825, 1998

[74] J. Jung, M. Perrut, Particle design using supercritical fluids : Literature and patent survey, J. Supercritical Fluids 20 (2001) 179-219

[75] G. Charbit, E. Badens, O. Boutin, Methods of particle production, dans Drug Delivery and Supercritical Fluid Technology, Marcel Dekker (ed.), sous presse

[76] A. Bertucco, P. Pallado, L. Benedetti, Formation of biocompatible polymer microspheres for controlled drug delivery by a supercritical antisolvent technique, Proceedings of the 3rd Symposium on High Pressure Chemical Engineering, Zürich, 1996, pp. 217-222

[77] P. Pallado, P.L. Benedetti, L. Callegaro, Nanospheres comprising a biocompatible polysaccharide, US Patent 6 214384,2001

[78] N. Elvassore, A. Bertucco, P. Caliceti, Production of insulin-Ioaded poly(ethylene gIycol)/poly(lactide) (PEG/PLA) nanoparticles by gas antisolvent techniques, J. Pharmaceutical Sciences 90 (2001) 1628-1636
[79] O.I. Corrigan, A.M. Crean, Comparative physicochemical properties of hydrocorisone-PVP composites prepared using supercritical carbon dioxide by the GAS anti-solvent recrystallization process, by coprecipitation and spray drying, Int. J. Pharmaceutics 245 (2002) $75-82$

[80] C. Bitz, E. Doelker, Influence of the preparation method on residual solvents in biodegradable microspheres, Int. J. Pharmaceutics 131 (1996) 171-181

[81] S. Taki, E. Badens, G. Charbit, Controlled release system formed by supercritical anti-solvent coprecipitation of a herbicide and a biodegradable polymer, J. Supercritical Fluids 21 (2001) 61-70

[82] H. Lochard, E. Rodier, M. Sauceau, J.J. Letourneau, B. Freiss, C. Joussot-Dubien, J. Fages, A three step supercritical process to improve the dissolution rate of Eflucimibe, Proceedings of the 6th International Symposium on Supercritical Fluids, Versailles, 2003, pp. 1659-1664

[83] M. Perrut, Supercritical fluids applications: Industrial developments and economic issues, Ind. Eng. Chem. 39 (2000) 4531-4535 